\title{
Role for protease activity in visceral pain in irritable bowel syndrome
}

\author{
Nicolas Cenac, ${ }^{1}$ Christopher N. Andrews, ${ }^{2}$ Marinella Holzhausen, ${ }^{1}$ Kevin Chapman, ${ }^{1}$ \\ Graeme Cottrell, ${ }^{3}$ Patricia Andrade-Gordon, ${ }^{4}$ Martin Steinhoff, 5 Giovanni Barbara, ${ }^{6}$ \\ Paul Beck, ${ }^{2}$ Nigel W. Bunnett, ${ }^{3}$ Keith A. Sharkey, ${ }^{7}$ Jose Geraldo P. Ferraz, ${ }^{2}$ \\ Eldon Shaffer, ${ }^{2}$ and Nathalie Vergnolle ${ }^{1}$
}

\begin{abstract}
${ }^{1}$ Department of Pharmacology and Therapeutics and 2Department of Medicine, Division of Gastroenterology, University of Calgary, Calgary, Alberta, Canada. ${ }^{3}$ Departments of Surgery and Physiology, UCSF, San Francisco, California, USA. ${ }^{4}$ R.W. Johnson Pharmaceutical Research Institute, Spring House, Pennsylvania, USA. ${ }^{5}$ Department of Dermatology and Interdisciplinary Center for Clinical Research (IZKF) Münster, University of Münster, Münster, Germany. ${ }^{6}$ Departments of Internal Medicine and Gastroenterology, University of Bologna, Bologna, Italy. ${ }^{7}$ Department of Physiology and Biophysics, Faculty of Medicine, University of Calgary, Calgary, Alberta, Canada.
\end{abstract}

\begin{abstract}
Mediators involved in the generation of symptoms in patients with irritable bowel syndrome (IBS) are poorly understood. Here we show that colonic biopsy samples from IBS patients release increased levels of proteolytic activity (arginine cleavage) compared to asymptomatic controls. This was dependent on the activation of $\mathrm{NF}-\kappa \mathrm{B}$. In addition, increased proteolytic activity was measured in vivo, in colonic washes from IBS compared with control patients. Trypsin and tryptase expression and release were increased in colonic biopsies from IBS patients compared with control subjects. Biopsies from IBS patients (but not controls) released mediators that sensitized murine sensory neurons in culture. Sensitization was prevented by a serine protease inhibitor and was absent in neurons lacking functional protease-activated receptor-2 $\left(\mathrm{PAR}_{2}\right)$. Supernatants from colonic biopsies of IBS patients, but not controls, also caused somatic and visceral hyperalgesia and allodynia in mice, when administered into the colon. These pronociceptive effects were inhibited by serine protease inhibitors and $\mathrm{PAR}_{2}$ antagonist and were absent in $\mathrm{PAR}_{2}$-deficient mice. Our study establishes that proteases are released in IBS and that they can directly stimulate sensory neurons and generate hypersensitivity symptoms through the activation of $\mathrm{PAR}_{2}$.
\end{abstract}

\section{Introduction}

Irritable bowel syndrome (IBS) is one of the most common gastrointestinal disorders, afflicting $10 \%-15 \%$ of the population in developed countries $(1,2)$. Considered a functional bowel disorder, IBS is characterized by abdominal discomfort and pain, associated with altered bowel function. IBS bowel symptoms may be characterized by a predominance of constipation (IBS-C) or diarrhea (IBS-D) or by alternating constipation and diarrhea (IBS-D/C). The notion of "functional" comes from an apparent absence of findings supporting an organic basis, since there are no biochemical, histopathological, or diagnostic imaging criteria yet characterized that define the syndrome. Accordingly, stress or affective disorders were long assumed to constitute the key mechanisms in the development of IBS $(3,4)$. Microscopic inflammation has recently been proposed to be associated with the development of IBS symptoms in some patients (5-8). A history of a gastrointestinal infectious episode is also associated with the development of IBS in $18 \%$ of these patients (9). IBS can also appear in patients with inflammatory bowel disease (IBD) who are in remission (10). A unifying hypothesis for the generation of symptoms of IBS incorporates a central component (psychological factors), together with peripheral organ dysfunction (motility disorders, visceral hypersensitivity), possibly aggravated by microscopic inflamma-

Nonstandard abbreviations used: DRG, dorsal root ganglion; gusb, $\beta$-glucuronidase; IBD, inflammatory bowel disease; IBS, irritable bowel syndrome; IBS-C, IBS with constipation; IBS-D, IBS with diarrhea; IBS-D/C, IBS with alternating constipation and diarrhea; $\mathrm{PAR}_{2}$, protease-activated receptor- 2 .

Conflict of interest: The authors have declared that no conflict of interest exists. Citation for this article: J. Clin. Invest. 117:636-647 (2007). doi:10.1172/JCI29255. tion and/or previous intestinal infection. Most IBS patients experience underlying visceral hyperalgesia, as measured in studies in which a balloon catheter inserted into the rectum was inflated and provoked an increased nociceptive response (hyperalgesia) and a decreased nociceptive threshold (allodynia) (11).

A major obstacle to the effective treatment of IBS is that the mediators of symptoms such as abdominal pain and their mechanisms of action are unknown. We sought to determine whether mediators released by colonic tissues from IBS patients can signal to sensory neurons and reproduce the hypersensitivity symptoms in animals. We focused our attention on proteases and their receptors, since they have been shown to cause visceral hyperalgesia in animal models $(12,13)$. Our results show that: (a) biopsies from IBS patients release elevated proteolytic activity compared with those from control patients; (b) this proteolytic activity can signal to sensory neurons through a mechanism involving the activation of protease-activated receptor-2 ( $\left.\mathrm{PAR}_{2}\right)$; and (c) proteolytic activity released from IBS patient tissues, when introduced into the colon of mice, causes hypersensitivity symptoms (hyperalgesia and allodynia) through a $\mathrm{PAR}_{2}$-dependent mechanism. The present study provides evidence for a crucial role for proteases released by colonic tissues of IBS patients as mediators that generate hypersensitivity symptoms.

\section{Results}

Colonic biopsies from IBS and IBD patients release proteolytic activity. Colonic tissue biopsies incubated in culture medium for 1 hour released proteolytic activity that cleaves at an arginine site, hydrolyzing the substrate tert-butyloxycarbonyl-Gln-Ala-Arg-7-amino4-methycoumarin (tert-butyloxycarbonyl-Gln-Ala-AMC) (Figure 1, A and B). Proteolytic activity released from IBS and IBD patient 

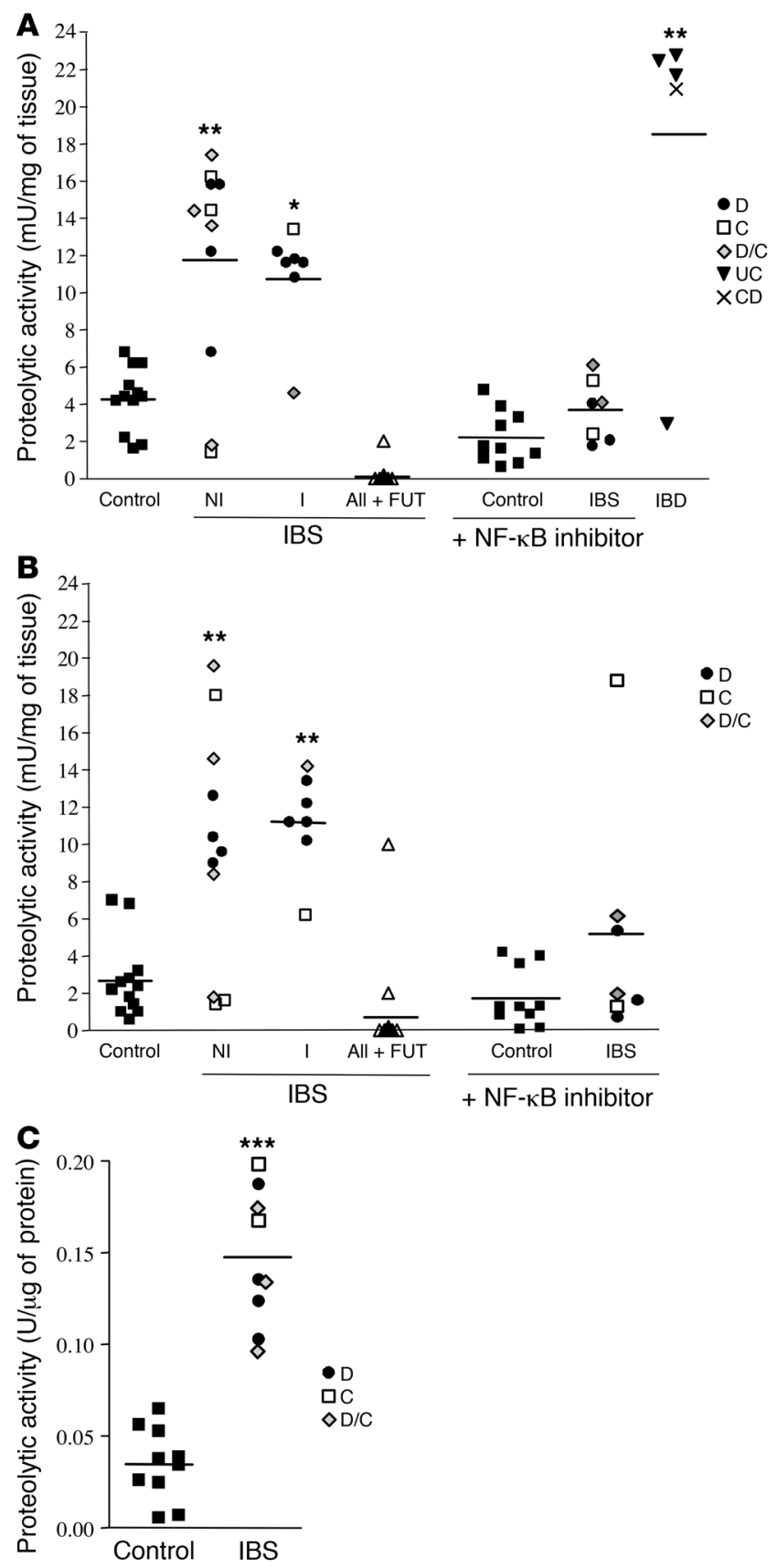

biopsies was, respectively, 2- to 3-fold and 6-fold higher than the activity in control patient samples. The serine protease inhibitor FUT-175 completely inhibited activity released from IBS and control biopsies (Figure 1, A and B). Similar levels of proteolytic activity were observed in supernatants of biopsies taken either from the ascending colon or the rectum of IBS patients (Figure 1, A and B). Incubation of with the NF- $\mathrm{B}$ inhibitor BAY 11-7085 (14) significantly reduced proteolytic activity released from IBS but not control biopsies (Figure 1, A and B). The levels of proteolytic activity detected in luminal washes of IBS patients were 5-fold higher than those detected in luminal washes of control patients (Figure 1C).

Trypsin and tryptase levels are increased in colonic biopsies from IBS patients, but mast cells numbers are not elevated. Tryptase and trypsin

\section{Figure 1}

Proteolytic activity in human colonic biopsies and present in colonic washes. Arginine-directed protease activity in supernatants of biopsy from controls (black squares), IBS patients (IBS-D: D, circles; IBS-C: $C$, white squares; or IBS-D/C: D/C, diamonds), IBD patients (ulcerative colitis [UC]: black triangles; Crohn disease [CD]: $x$ 's) or in IBS biopsy supernatants preincubated with the serine protease inhibitor FUT-175 or supernatants from biopsies incubated with the NF-kB inhibitor BAY 11-7085. IBS biopsy supernatants were separated according to the presence (I) or not (NI) of inflammatory signs, and biopsies were harvested at the level of the ascending colon $(\mathbf{A})$ or the rectum (B). All + FUT, all IBS patient supernatants (I and NI) incubated in the presence of FUT-175. (C) Protease activity in colonic washes from control (black squares) and IBS patients. Data are mean \pm SEM. ${ }^{*} P<0.05$, ${ }^{\star *} P<0.01,{ }^{* \star *} P<0.005$ compared with control group.

mRNA levels were quantified in colonic biopsies by semiquantitative RT-PCR (Figure 2A). A significant increase in tryptase and trypsin mRNA expression was observed in tissues from IBS patients compared with healthy controls, with a 1.8- to 2-fold increase for tryptase and a 2.2- to 2.8-fold increase for trypsin (Figure 2A). Similar increases in tryptase and trypsin mRNA expression were observed whether samples were taken from the rectum or ascending colon in IBS patients. Tryptase and trypsin proteins were released by biopsies from IBS and control patients, as detected by Western blotting. Densitometric analysis indicated that trypsin levels were 1.6- to 2 -fold and tryptase levels 1.8-fold higher in IBS biopsy supernatants than in controls (Figure 2B). In IBD patients, the profile of proteases released appears to be different from that in IBS patients. The level of trypsin protein released by IBD patient biopsies compared with control biopsies was not significantly increased. Only tryptase protein levels were higher, by 2 -fold, in IBD compared with control biopsy supernatants (Figure 2B).

Mast cells are important cellular sources of proteolytic enzymes including tryptase and tryptic-like enzymes (15-17). We therefore assessed the expression of tryptase immunoreactivity and the number of mast cells in the biopsy samples. Tryptase was mostly localized to cells found throughout the lamina propria (Figure 3) and around the base of rectal mucosal crypts. Based on their size and distribution, these cells are likely to be mast cells (17). Two other much smaller populations of cells were observed in these samples. Some cells (e.g., Figure 3C) had obvious processes at the apical and/or basal poles, and often these cells displayed intense immunoreactivity compared with neighboring cells. Occasional tryptase-immunoreactive cells were found in the epithelium of both control and IBS patients; these had the appearance of enteroendocrine cells (Figure 3D). No differences were observed between any of the cell populations in IBS and control patients with respect to either the density of cells or intensity of immunoreactivity (Figure 3G). As noted in Methods, tryptase immunoreactivity was found in some obviously degranulating cells. These cells were observed in both patient groups, and no obvious differences were noted, but these cells were not readily counted because of the diffuse nature of the immunoreactivity.

We also investigated the presence of mast cells using Alcian blue staining in the lamina propria of biopsy samples from IBS $(n=15)$ and control patients $(n=7)$. In IBS patient biopsies, there were no significant differences in the number of mast cells in the ascending colon $(21.3 \pm 3.1$ cells per $\times 10$ visual field $)$ and rectum $(13.7 \pm 3.1$ cells). Similarly, there were no differences in the number of mast 

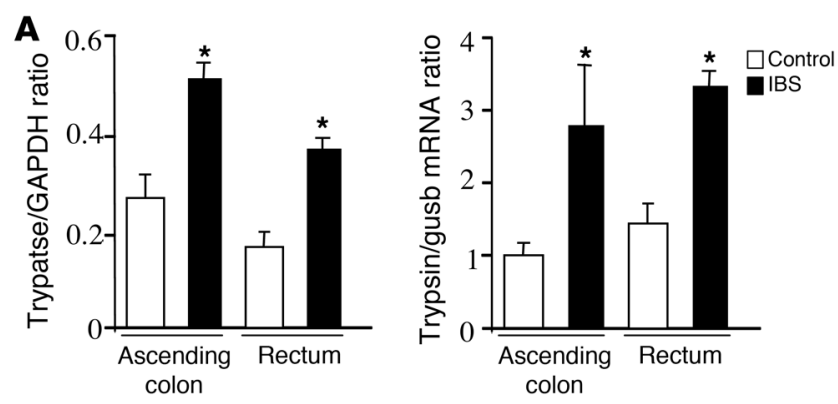

B
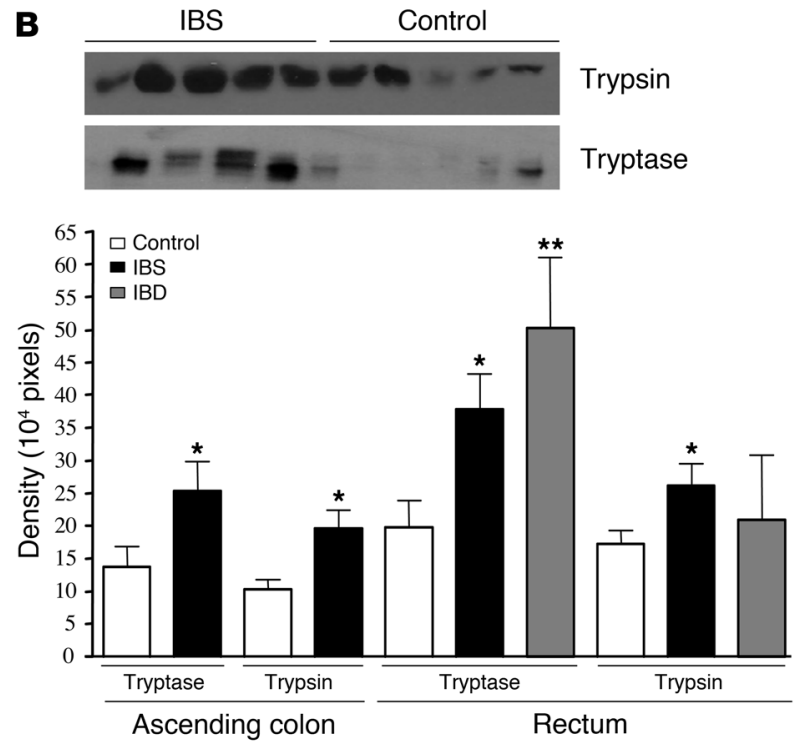

cells in control patients $(20.9 \pm 8.6$, rectum; $20.3 \pm 8.9$, ascending colon), and there were no differences between IBS patients and controls (Figure 3, E and F).

The levels of protease inbibitor $\alpha$-1-antiproteinase expression are unchanged in IBS and control patient biopsy supernatants, and enterokinase is detected only in IBS patient biopsy supernatants. The protein expression level of $\alpha$-1-antiproteinase (a trypsin, tryptase, and neutrophil elastase inhibitor) was evaluated in supernatants from IBS or control biopsies by Western blot analysis and subsequent gel densitometry. The mean expression of $\alpha$-1-antiproteinase was not significantly different in supernatants of IBS biopsies compared with supernatants of control biopsies $\left(268 \times 10^{3} \pm 39 \times 10^{3}\right.$ pixels versus $282 \times 10^{3} \pm 40 \times 10^{3}$ pixels; $n=10$ in each group).

The expression of enterokinase, which converts trypsinogen to trypsin (18) was also investigated in the supernatants from IBS or control biopsies by Western blot analysis. The presence of enterokinase was detected in only 2 of 10 supernatants from IBS patient biopsies but in none of the supernatants from control patient biopsies (data not shown).

IBS tissue supernatants activate sensory neurons: a protease and $P A R_{2}-$ dependent effect. To determine whether biopsies express mediators that can signal to sensory nerves, we examined the effects of biopsy supernatants on calcium mobilization of mouse dorsal root ganglion (DRG) neurons in culture. All supernatants from IBS biopsies induced a prompt increase in $\left[\mathrm{Ca}^{2+}\right]_{i}$ that was significantly higher than the signal caused by control patient supernatants (Figure 4). Supernatants from all the subgroups of IBS patients (IBS-D, IBS-C, IBS-D/C, or those with microscopic

\section{Figure 2}

Tryptase and trypsin expression in human colonic biopsies and culture supernatants. (A) Dual RT-PCR of tryptase and GADPH mRNA and of trypsin and gusb from IBS and control patient biopsies quantified by densitometry analysis of the gels. (B) Western blots of trypsin and tryptase proteins from control, IBS, or IBD patient biopsy supernatants (top panel), quantified by densitometry analysis of the blots (bottom panel). Data are mean $\pm \mathrm{SEM} ; n=12 .{ }^{*} P<0.05$ compared with control group; ${ }^{*} P<0.01$.

inflammation) evoked a similar increase in $\left[\mathrm{Ca}^{2+}\right]_{\mathrm{i}}$ compared with supernatants from healthy control patients (Figure 4). Similarly, increased responses of sensory neurons to IBS biopsy supernatants was observed whether biopsy supernatants were taken from the ascending colon (Figure 4A) or the rectum (Figure 4B).

In order to assess whether the response was due to proteolytic activity in the supernatants, we repeated the experiments from the IBS patients in the presence of the broad-spectrum serineprotease inhibitor FUT-175. The addition of FUT-175 to biopsy supernatants abolished the effects of IBS supernatants on $\left[\mathrm{Ca}^{2+}\right]_{i}$ in DRG neurons (Figure 4). Purified human trypsin and tryptase were also able to activate calcium mobilization in those cells, and this effect was blocked by FUT-175 (data not shown). In order to address the mechanism of biopsy supernatant-induced calcium mobilization, we isolated DRG neurons from mice lacking $\mathrm{PAR}_{2}$, as previous studies showed that $\mathrm{PAR}_{2}$ can play an important role in calcium signaling in response to proteases. We observed no increase in calcium mobilization in DRG neurons isolated from $\mathrm{PAR}_{2}$-deficient $\left(\mathrm{PAR}_{2^{--}}\right)$mice and exposed to IBS biopsy supernatants (Figure 4). Taken together, these results show that proteases released by colonic tissues of IBS patients can signal to sensory neurons by activating $\mathrm{PAR}_{2}$.

IBS patient tissue supernatants cause thermal and mechanical byperalgesia and allodynia in mice. Intraplantar administration of supernatants from IBS patients in mice decreased the withdrawal latency in response to thermal stimulus (Figure 5A), characteristic of thermal hyperalgesia, while supernatants from control patients had no effect. Similarly, IBS but not control patient biopsy supernatants injected into the mouse paw provoked a significant increase in nociceptive score in response to mechanical stimulation with von Frey filaments of various sizes (Figure 5, B-D). An increased nociceptive score was observed in response to innocuous stimulus (smallest filament: 3.61 ) in mice that had received in the paw supernatants from IBS patients, while a score of 0 was observed for all other groups (Figure 5B). This demonstrates that IBS supernatants caused allodynia. In response to noxious stimulus (larger filaments: 3.84 and 4.08), an increased nociceptive score was observed in IBS-injected mice compared with control supernatant-injected mice, demonstrating mechanical hyperalgesia. When injected into the paw of $P A R_{2}^{-1-}$ mice, IBS patient biopsy supernatants did not affect withdrawal latency (Figure 5A) or nociceptive score (Figure 5, B-D). These results point to a role for $\mathrm{PAR}_{2}$ activation in IBS patient biopsy supernatant-induced thermal and mechanical somatic hyperalgesia.

IBS tissue supernatants cause visceral bypersensitivity in mice: a protease and $P A R_{2}$-dependent effect. Intracolonic administration of supernatants from IBS patients increased the intensity of abdominal contractions in response to colorectal distension (Figure 6, A-C). From 6 to 12 hours after intracolonic administration of supernatants from IBS patients, the intensity of abdominal contractions 

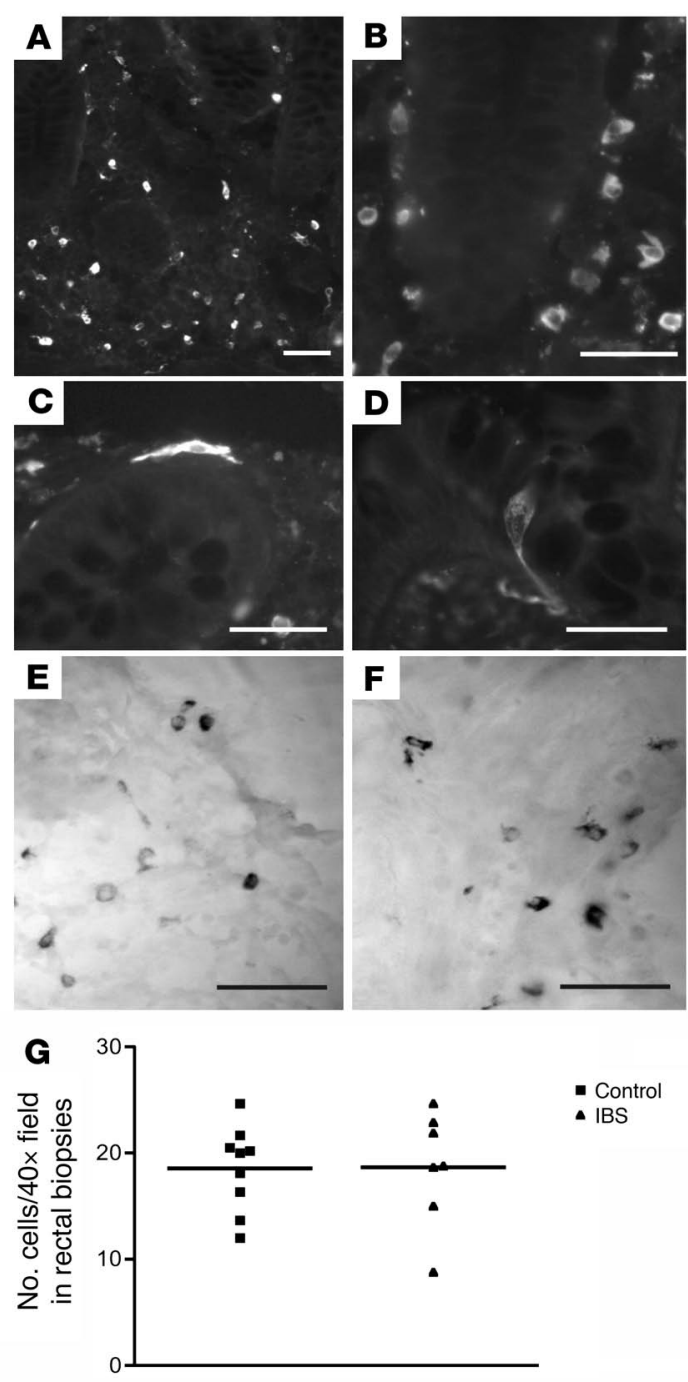

in response to different pressures of colorectal distension (15-60 $\mathrm{mmHg}$ ) was significantly increased compared with values measured before the intracolonic treatment (time 0) (Figure 6B). Increased intensity of abdominal contractions after IBS supernatant treatment was observed in response to innocuous $(15 \mathrm{mmHg})$ or noxious $(30-60 \mathrm{mmHg}$ ) stimuli, demonstrating the occurrence of both allodynia and hyperalgesia. In contrast, mice that had received intracolonic treatments with supernatants from control patients demonstrated levels of abdominal contraction response similar to those observed before the intracolonic administration (Figure 6C, far-left panel, triangles). When the protease inhibitor FUT-175 was added to biopsy supernatants from IBS patients, those supernatants did not elicit the colorectal distension-induced increase in abdominal contractions (Figure 6C, second panel). $P A R_{2}{ }^{-/-}$mice, or mice treated with a $\mathrm{PAR}_{2}$ antagonist (19), did not demonstrate IBS supernatant-induced increases in abdominal contractions compared with basal distension (before intracolonic administration) (Figure 6C, third and fourth panels, respectively).

Supernatants from all the subgroups of IBS patients (IBS-D, IBS-C, IBS-D/C, and those with microscopic inflammation) evoked a similar increase in the intensity of abdominal contraction in response to colorectal distension at all pressures compared

\section{Figure 3}

Tryptase immunoreactivity in rectal biopsies from control (A, D, and E) and IBS patients (B, C , and F). Tryptase was mostly localized to cells found throughout the lamina propria $(\mathbf{A}-\mathbf{C})$ and around the base of rectal mucosal crypts (C). Some cells (e.g., C), had obvious processes at either the apical and/or basal poles, and often these cells displayed intense immunoreactivity compared with neighboring cells (images in $\mathbf{B}$ and $\mathbf{C}$ were taken under identical exposure conditions and reflect differences in intensity). Occasional tryptase-immunoreactive cells were found in the epithelium of both control (D) and IBS patients; these had the appearance of enteroendocrine cells, based on the obvious basolateral process. No differences were observed in the cell populations of IBS and control patients with respect to either the density of cells in rectal biopsies (G) or the intensity of immunoreactivity. Alcian blue-labeled mast cells in a control (E) and IBS patient (F). Note that the majority of tryptase-immunoreactive cells (e.g., B) have the size, shape, and cellular location of mast cells. Scale bars: $50 \mu \mathrm{m}$.

with control distension (before intracolonic administration of supernatant) (Figure 7).

The results shown in Figures 6 and 7 were obtained using supernatants from biopsies harvested from the ascending colon, but similar results were observed with supernatants of biopsies from the rectum (data not shown). Intracolonic administration of $0.5 \mathrm{U}$ of trypsin $(100 \mu \mathrm{l})$ increased the intensity of abdominal contraction in response to colorectal distension at all pressures (Figure 8). However, this increase was not observed after the administration of $0.2 \mathrm{U}$ of trypsin (Figure 8). Interestingly, $0.52 \mathrm{U}$ of trypsin corresponds to the average proteolytic activity released from IBS biopsies and present in $100 \mu \mathrm{l}$ of supernatants that were injected to mice. $0.2 \mathrm{U}$ of trypsin corresponds to the average proteolytic activity in control biopsies (Figure 8).

\section{Discussion}

We show that proteolytic at an arginine site is released in significantly higher amounts from colonic biopsies of IBS and IBD patients compared with controls. Moreover, proteolytic activity released in vivo in the lumen of IBS patients in significantly higher quantities than in controls. Further, we show that this proteolytic activity is responsible for activation of sensory neurons, as well as generation of somatic hyperalgesia and visceral hypersensitivity, through a mechanism involving the activation of $\mathrm{PAR}_{2}$. These results are in accordance with the concept that $\mathrm{PAR}_{2}$ represents an important receptor mediating the symptoms of IBS (20). Thus, $\mathrm{PAR}_{2}$ activation modifies intestinal chloride secretion, consistent with a role for this receptor in IBS-associated secretory dysfunctions (21-24). $\mathrm{PAR}_{2}$ agonists evoke an increase in intestinal barrier permeability $(25,26)$, which is known to be associated with IBS symptoms in some patients $(27,28)$ as well as in animal models (29). PAR $_{2}$ activation in the colon of mice induces signs of inflammation $(20,25)$, while the putative role for microinflammation in tissues of IBS patients is now widely recognized (5-7). $\mathrm{PAR}_{2}$ activation also modifies gastrointestinal motility patterns in vivo and in vitro (30-32). But the strongest evidence for a role for $\mathrm{PAR}_{2}$ in generation of IBS symptoms relies on the effects of $\mathrm{PAR}_{2}$ activation on visceral nociceptive functions. It has been established that $\mathrm{PAR}_{2}$ agonists evoke long-lasting visceral hypersensitivity (12) and hyperexcitability of enteric neurons $(33,34)$. In the present study, we shed light on the role of $\mathrm{PAR}_{2}$ in human pathophysiology, demonstrating that proteases that can signal through $\mathrm{PAR}_{2}$ are specifi- 

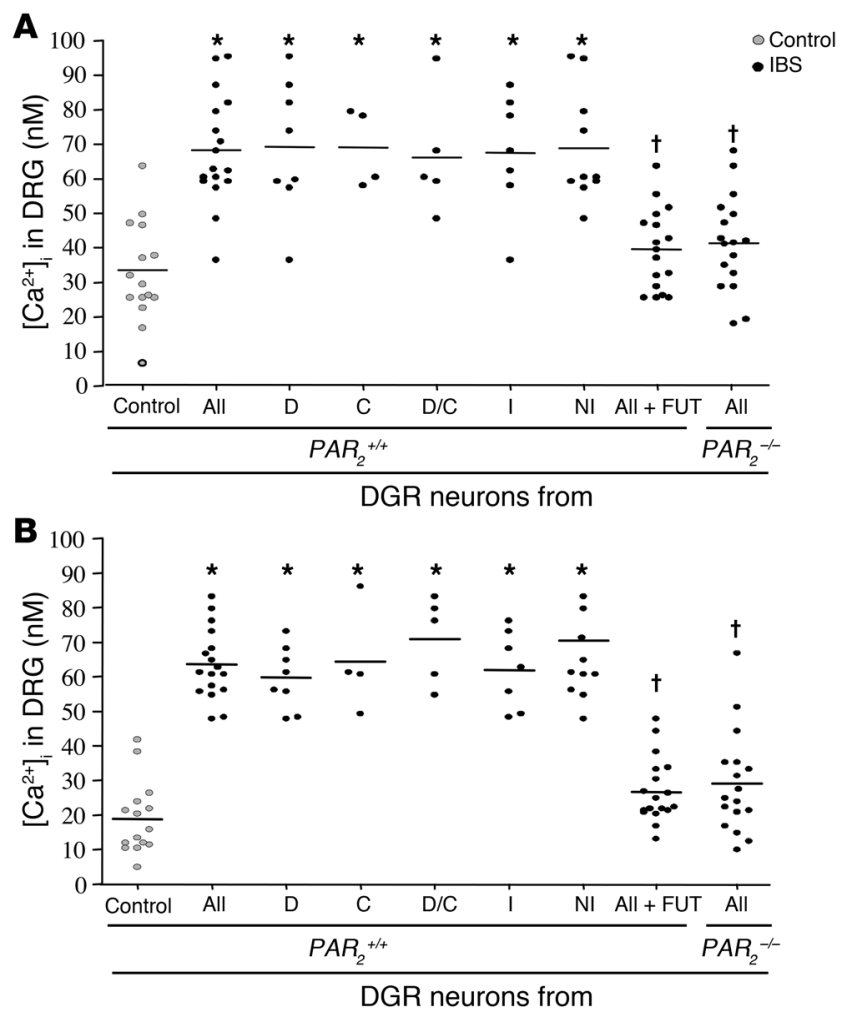

cally released from colonic biopsies of IBS patients. Moreover, we show here that of all the mediators released from these biopsies, proteases play a major role in signaling to sensory nerves and in the generation of visceral hypersensitivity, since the serine-protease inhibitor FUT-175 abolished calcium mobilization in mouse DRG neurons and signs of visceral pain. The use of $P A R_{2}^{-1-}$ mice and a $\mathrm{PAR}_{2}$ antagonist clearly revealed that these responses are dependent on the activation of $\mathrm{PAR}_{2}$. We have also observed increased proteolytic activity in biopsies from IBD patients. $\mathrm{PAR}_{2}$, which is known to mediate the proinflammatory effects of trypsin and tryptase (35), is also overexpressed in tissues from IBD patients (36). This suggests that $\mathrm{PAR}_{2}$ could be a common target in the treatment of IBD and IBS. The fact that proteolytic activity is increased in both IBS and IBD patient biopsy supernatants demonstrates that high proteolytic activity is not specifically associated with IBS but is also involved in other gastrointestinal pathologies. However, the increased trypsin expression seems to be specific to IBS, as it was not observed in IBD biopsy supernatants.

Among the mediators that are present and potentially released from tissues of IBS patients, it was surprising to see that proteases accounted for almost all of the pronociceptive effects of IBS biopsy supernatants: hyperalgesia, allodynia, and the calcium flux in sensory neurons were abolished by protease inhibitor FUT-175. Other mediators, such as 5-hydroxytryptamine (5-HT), histamine, ATP, or prostaglandins, which are likely to be present and released by mucosal biopsies, can signal to sensory nerves and have been shown to induce visceral hypersensitivity $(37,38)$. One possible explanation for these data is that proteases are less susceptible to degradation than other mediators in our experimental conditions of incubation and therefore account for most of the pronociceptive effects. However, it may also be hypothesized that proteases

\section{Figure 4}

Calcium mobilization in sensory neurons exposed to human colonic biopsy supernatants. Calcium flux in sensory neurons from wild-type $\left(P A R_{2}{ }^{+/+}\right)$and $P A R_{2}^{-/-}$mice exposed to supernatants of biopsies from control and IBS patients or biopsy supernatants preincubated with a serine protease inhibitor (FUT-175). IBS patient supernatants were either regrouped together (all supernatants [All]) or divided into subgroups. Biopsies were collected either from the ascending colon $(\mathbf{A})$ or the rectum (B). Data are mean \pm SEM. ${ }^{*} P<0.05$ compared with control group; $\uparrow P<0.05$ compared with the group with all IBS supernatants tested in $P A R 2^{+/+}$neurons.

play a pivotal role in sensitization of sensory nerves to other pronociceptive mediators. This hypothesis is consistent with the finding that activation of the calcium channel TRPV1 is potentiated by preexposure to $\mathrm{PAR}_{2}$-activating proteases $(39,40)$.

The question of the nature of the proteases that are specifically released from IBS patient biopsies is essential to the identification of potential new targets for the treatment of IBS. Our results show that the proteases tryptase and trypsin, both of which can activate $\mathrm{PAR}_{2}$, are upregulated and released into biopsy supernatants. This is in agreement with previous results that have demonstrated that the mucosal content of tryptase was increased in IBS patients and that the number of degranulating mast cells was increased by $150 \%$ in the same patients (41). In our study, we did not observe an increase in the number of mast cells present in the biopsies from controls or IBS patients, but we detected a significant increase in the amount of tryptase released in IBS compared with control biopsy supernatants. This suggests, like the study by Barbara et al. $(41,42)$, that although higher mast cell numbers might not be present in tissues, more mast cells are actively degranulating in tissues of IBS patients. Other studies, such as that by Weston et al., have shown an increase in mast cell numbers in ileal tissues of IBS compared with control patients (43). Several reasons could be given to explain the discrepancies among all the studies on mast cell numbers in IBS patient biopsies, including the counting methods, the nature of the biopsies and the regions of the gut that were sampled, and the detection methods employed. The overall conclusion of all those studies is that mast cell mediators, and particularly tryptase, are released in significantly higher amounts in the tissues of IBS patients. In their study, Barbara et al. demonstrated that in IBS patients, mast cells lie closer to nerve fibers of the mucosa compared with those in control patients (42). This suggests that once released by degranulating mast cells, tryptase could be in direct contact with enteric or primary afferent nerves, both of which express $\mathrm{PAR}_{2}(5-7,33,44$, $45)$, and induce hyperexcitability $(34,45)$.

Nonpancreatic trypsin can be released from different cell types of the gut, including endothelial cells, and epithelial cells (46). In a recent report, we demonstrated that upon bacterial infection, colonic tissues from the infected mouse host were able to express significant amounts of trypsin, which then participated in the generation of an inflammatory response through the activation of $\mathrm{PAR}_{2}$ (47). Therefore, both trypsin and tryptase can be considered as the proteases released from IBS patient tissues that are responsible for $\mathrm{PAR}_{2}$ activation. Cells that produce tryptase (mostly mast cells) and trypsin (endothelial and/or epithelial cells) could act as effectors that release their mediators (proteases), which then generate action potential in enteric and primary afferent nerve endings within the lamina propria, leading to enteric neuron hyperexcitability and visceral hyperalgesia. It is interesting to note that 

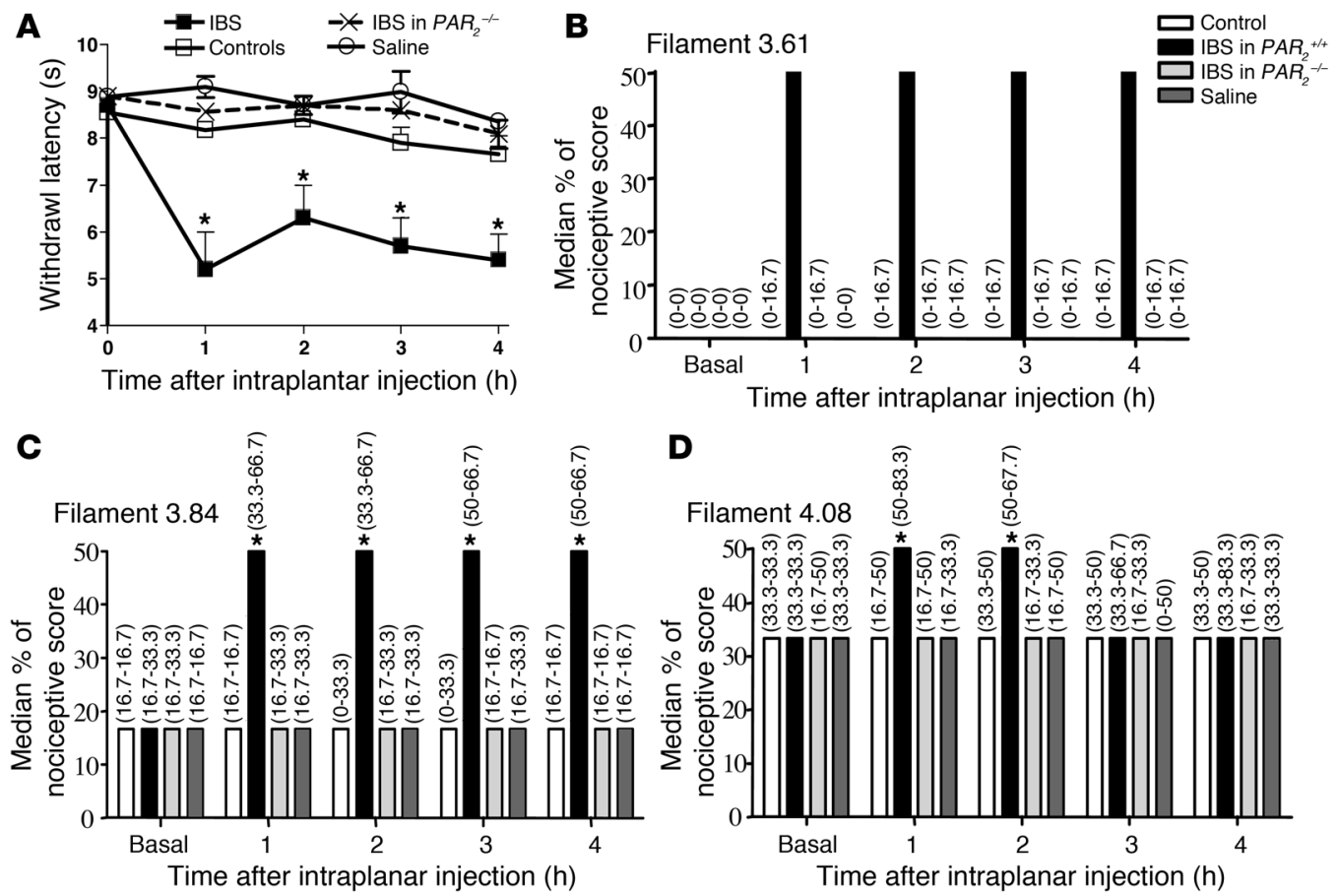

Figure 5

Somatic nociception of mice in response to injection of human colonic biopsy supernatants into paws. Graph of withdrawal latency (A) and nociceptive scores (B-D) in response to thermal (plantar test) or mechanical (von Frey filaments) stimulation, respectively, of mouse paws that had received an injection of control or IBS patient biopsy supernatants or saline solution. Wild-type mice $\left(P A R_{2}^{+/+}\right.$: squares [A], black bars [B-D]) and $\mathrm{PAR}_{2}$-deficient mice $\left(P A R_{2}^{-l}: x^{\prime}\right.$ 's [A], gray bars [B-D]) had received intraplantar injections. Three sizes of von Frey filaments were used: filament 3.61 (B), filament 3.84 (C), and filament 4.08 (D). All biopsies represented in this figure were collected from the rectum; $n=8$ for controls; $n=9$ for IBS. Data are mean \pm SEM for withdrawal latencies and median and range (in brackets) for mechanical stimuli testing (von Frey filaments). ${ }^{\star} P<0.05$ compared with basal measures (before the intraplantar injection: time 0 ).

the amount of proteolytic activity in IBS patient biopsies, when compared to the activity of known concentrations of trypsin, is perfectly within the range of trypsin concentrations that are necessary to activate $\mathrm{PAR}_{2}$ on sensory neurons (48). Moreover, the dose of trypsin $(0.2 \mathrm{U})$ that corresponds to the average proteolytic activity in control biopsies had no effect on colonic sensitivity to colorectal distension. In contrast, doses of trypsin that correspond to the proteolytic activity released by IBS biopsies provoked allodynia and visceral hyperalgesia (Figure 8). Why IBS should be associated with increased proteolytic activity in the intestinal mucosa remains to be clarified. Another possible source of proteases could be the luminal microflora. Several studies have suggested that bacterial flora is different in IBS and control patients $(49,50)$. Our study shows that trypsin and tryptase transcription in IBS patient tissues is increased, suggesting that those proteases are at least in part responsible for the IBS-associated increased proteolytic activity. However, we cannot rule out the possibility that bacterial serine proteases could also be partly responsible for increased mucosal proteolytic activity. Further studies could investigate proteolytic activity in tissues of patients receiving treatments that would affect their microflora (probiotic or antibiotic treatments) or their pancreatic enzyme secretion (atropine treatments).

We observed that the expression of $\alpha$-1-antiproteinase, one of the most studied inhibitors of trypsin, tryptase, and neutrophil elastase, was not different in supernatants of biopsies from control and IBS patients. This finding, although limited to one member of the serpin family, argues against the hypothesis that decreased expression of protease inhibitor could allow increased proteolytic activity at mucosal surfaces of IBS patients. The presence of enterokinase in supernatants of some IBS patient biopsies suggests that pro-forms of enzymes, in particular trypsinogen, could be more effectively cleaved into active enzymes in tissues of IBS patients. However, the proportion of IBS patient tissues that express enterokinase is relatively small ( 2 of 10 patients), suggesting that the presence of enterokinase in IBS patient tissues could not be the sole mechanism to explain IBS-associated increased activation of proteases. Finally, we have observed that incubation of biopsies in the presence of a specific NF-кB inhibitor (14) significantly decreased the proteolytic activity released by IBS but not control patient biopsies (Figure 1, A and B). This result could suggest that $\mathrm{NF}-\kappa \mathrm{B}-$ dependent transcription of proteases is increased in IBS patient tissues. In fact, trypsin and tryptase mRNA levels were significantly increased in IBS patient biopsies (Figure 2). Although several studies demonstrated that arginine-directed proteases can activate NF- $\mathrm{KB}(51-54)$, the regulation of serine protease secretion

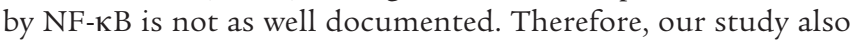
suggests that arginine-directed protease expression and activity, as has been demonstrated for matrix metalloproteinases (55-57), depends on NF-кB DNA binding.

In the visceral hypersensitivity experiments, IBS patient biopsy supernatants induced hyperalgesia and allodynia only several hours (6 hours; Figure 6A) after intracolonic administration. In 


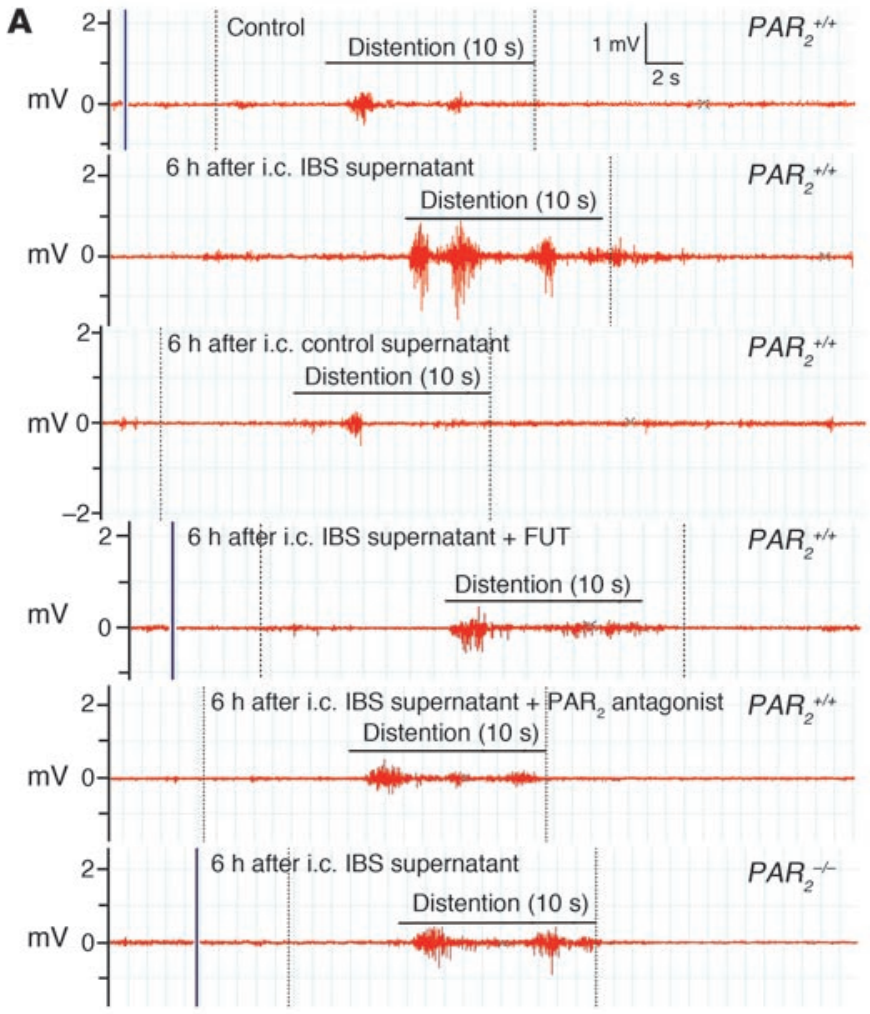

B
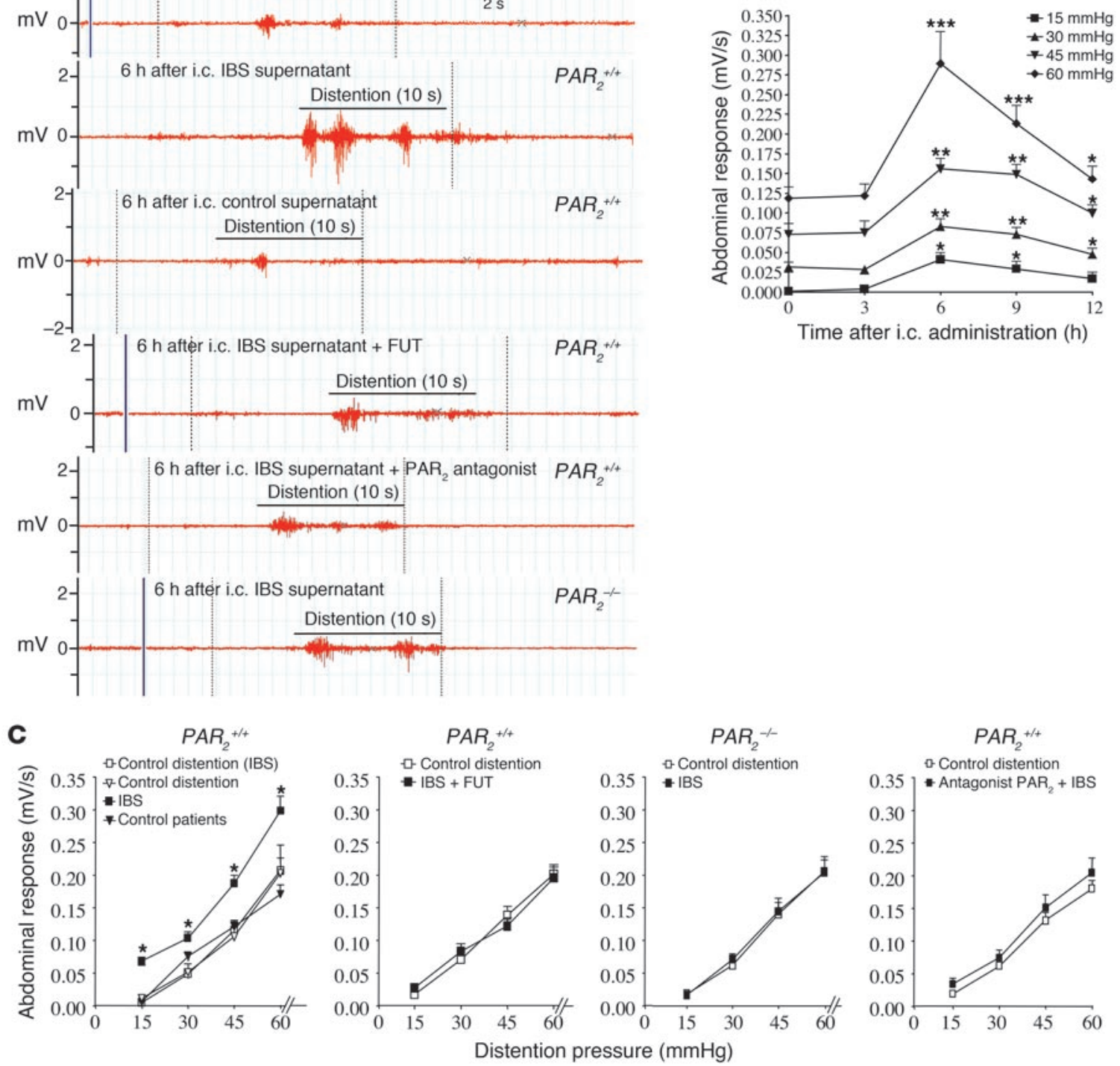

Figure 6

Visceral sensitivity in response to human colonic biopsy supernatants. Recordings of mouse abdominal muscle contraction in response to colorectal distension before (control) or 6 hours after intracolonic administration of ascending colon biopsy supernatants from IBS patients (A and $\mathbf{B}$ ) or control patients (filled triangles in $\mathbf{C}$ ). (A) Representative traces of mouse abdominal muscle contractions in response to $60 \mathrm{mmHg}$ colorectal distension, in $P A R_{2}{ }^{+/+}$or $P A R_{2}{ }^{-/}$mice before (control) or after intracolonic (i.c.) administration of IBS ascending colon biopsy supernatant alone or in combination with the protease inhibitor FUT or a $\mathrm{PAR}_{2}$ antagonist. (B) Abdominal contraction responses to different pressures of distension (15-60 mm $\mathrm{mg}$ ) before (time 0 ) or after intracolonic administration of IBS patient ascending colon biopsy supernatants. Data are mean \pm SEM; $n=8 .{ }^{\star} P<0.05,{ }^{* \star} P<0.01$, ${ }^{* *} P<0.005$ compared with basal (time 0 ) measurements. (C) Mouse abdominal contraction response to increasing pressures of distension in $P A R_{2}{ }^{+/+}$or $P A R_{2}{ }^{-/}$mice before (control distension) or 6 hours after intracolonic administration

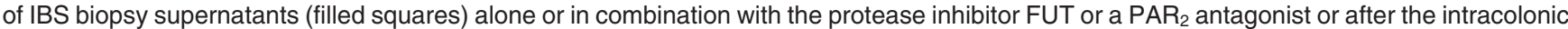
administration of biopsy supernatants from control patients (filled triangles). Data are mean $\pm \mathrm{SEM} ; n=12$ for the biopsy supernatants from control patients; $n=18$ for IBS biopsy supernatants. ${ }^{*} P<0.05$, ${ }^{* \star} P<0.01$, ${ }^{* *} P<0.005$ compared with control distension. For all data in this figure, biopsies were collected from the ascending colon.

contrast, the effects of the same supernatants on calcium signaling in DRG neurons were observed within seconds, and the pronociceptive effects in the paw (Figure 5) were significant before the first hour. This delay in the appearance of visceral hyperalgesia but not somatic hyperalgesia could be explained by the mode of administration of the supernatants. Intraluminal administration requires that the mediators penetrate the tissues, crossing the epithelial barrier, which might delay the effects of those mediators on primary afferents. It has been established that intracolonic administration of $\mathrm{PAR}_{2}$ agonists caused a breakdown in the integrity of the intestinal barrier $(25,26)$. Therefore, the action of $\mathrm{PAR}_{2}$-activating proteases administered in the mouse colonic lumen in the setting 


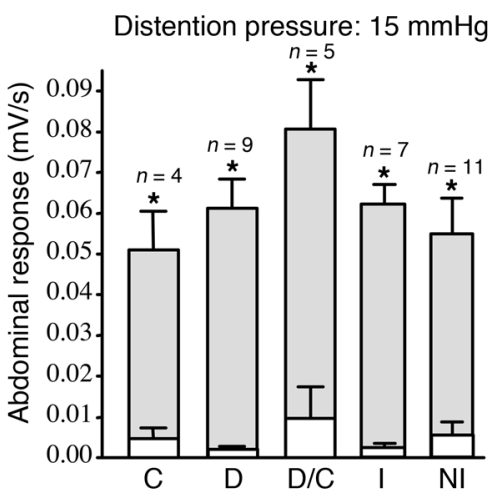

Distention pressure: $45 \mathrm{mmHg}$

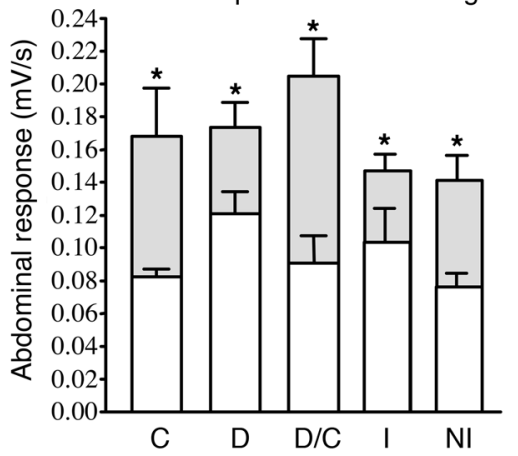

Distention pressure: $30 \mathrm{mmHg}$

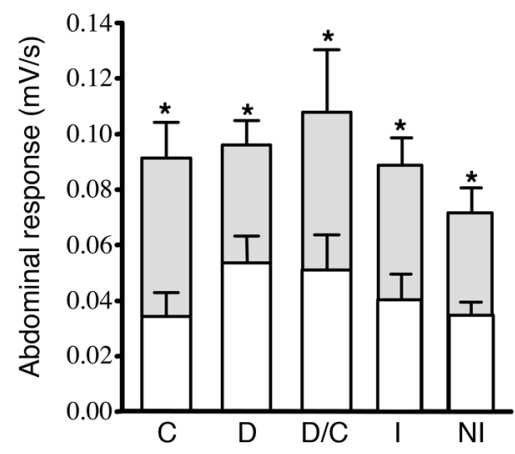

Distention pressure: $60 \mathrm{mmHg}$

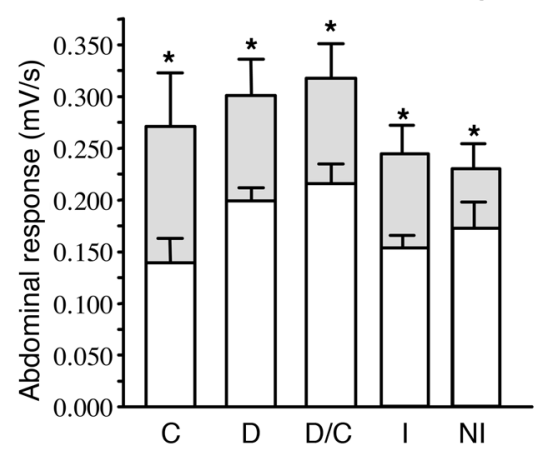

Figure 7

Visceral sensitivity in response to human colonic biopsy supernatants from different IBS patient subgroups. Abdominal muscle contraction responses of mice before (white bars) and 6 hours after intracoIonic administration of IBS ascending colon biopsy supernatant (gray bars). Results were analyzed in different subgroups: IBS-C, IBS-D, IBS-D/C, inflammation, and no inflammation. Data are mean \pm SEM. ${ }^{*} P<0.05$ compared with control distension.

mon therapeutic targets in all IBS patients for the treatment of abdominal pain.

\section{Methods}

Patients and biopsies. Patients with IBS, as defined by the Rome II criteria $(59,60)$, undergoing clinical evaluation at the University of Calgary gastrointestinal (GI) clinic were invited to participate in the study (Tables 1 and 2). Patients with confirmed diagnosis of celiac disease, infectious colitis, endocrine or CNS disorders, or GI malignancy were excluded. IBD patients with a confirmed diagnosis of Crohn disease or ulcerative colitis who were undergoing colonoscopy for assessment of disease activity were also invited to participate. Individuals undergoing colorectal cancer screening had tissue samples collected during colonoscopy and were considered controls (see Tables 1 and 2). The University of Calgary Conjoint

of visceral hypersensitivity might be primarily on intestinal permeability and then secondarily on primary afferent nerves.

Similar calcium signals in DRG neurons (Figure 4) and visceral hypersensitivity responses (Figure 7) were observed whether the biopsies were collected from IBS-D, IBS-C, or IBS-D/C patients. Moreover, the presence of microscopic inflammation in some IBS patients did not modify the responses observed in vivo or in vitro. We did not expect to find a common pattern of responses in these tissues that would suggest common mediators (proteases and $\mathrm{PAR}_{2}$ ) in all IBS patients, despite the variability in clinical presentation. Whether $\mathrm{PAR}_{2}$ and proteases contribute to altered bowel frequency and stool consistency is not known, but the fact that $\mathrm{PAR}_{2}$ activation leads to changes in intrinsic and extrinsic primary afferents $(33,34,45,58)$ is not inconsistent with these findings, since alterations in sensory processing in the gut wall could just as easily lead to enhanced as reduced motility, for example. Since similar findings for $\mathrm{PAR}_{2}$ and proteolytic activity were observed regardless of the IBS subtype, we cannot exclude the possibility that $\mathrm{PAR}_{2}$ activation and protease release could be a feature common to all colonic pathologies associated with hyperalgesia. However, our results strongly suggest a common function for $\mathrm{PAR}_{2}$-activating proteases on a clinical feature reported in all IBS patients: visceral hypersensitivity. Importantly, our results highlight $\mathrm{PAR}_{2}$-activating proteases and $\mathrm{PAR}_{2}$ as potential com-

\section{Figure 8}

Visceral sensitivity in response to different doses of trypsin. Abdominal contraction response of mice 6 hours after intracolonic administration of trypsin $(0.2,0.5$, and $1.0 \mathrm{U}$ of trypsin) or its vehicle (saline) in response to different pressures of distension $(15-60 \mathrm{mmHg}$ ). Data are mean $\pm \mathrm{SEM} ; n=8$ per dose of trypsin. ${ }^{\star} P<0.05,{ }^{\star \star} P<0.01$, ${ }^{\star * \star} P<0.005$ compared with control distension (time 0 ).
Ethics Committee approved the human research protocol. Written and verbal informed consent was obtained prior to enrollment in the study, according to the Declaration of Helsinki. The colonoscopic procedure employed a standard bowel preparation with an osmotic lavage (polyethylene glycol in the form of Colyte; GlaxoSmithKline), 3 days of clear fluids, and an overnight fast. To achieve conscious sedation, the narcotic fentanyl (Sublimaze; Abbott) and a benzodiazepine, diazepam in lipid emulsion (Diazemuls; Pfizer) or midazolam (Versed; Novopharm Ltd.), were administered intravenously. All colonic biopsies were collected during intubation of the colon and were taken first from the rectum (to avoid any artifact of intubation trauma) and then from the ascending colon.

Eight biopsies (10-20 mg) were collected from each control, IBS patient, or IBD patient: 4 biopsies from the rectum and 4 from the ascending colon. Fresh biopsies were rinsed in saline and incubated immediately in $2 \mathrm{ml}$ of HBSS at $37^{\circ} \mathrm{C}$ for 1 hour. Biopsies of the second group of patients (Table 2)

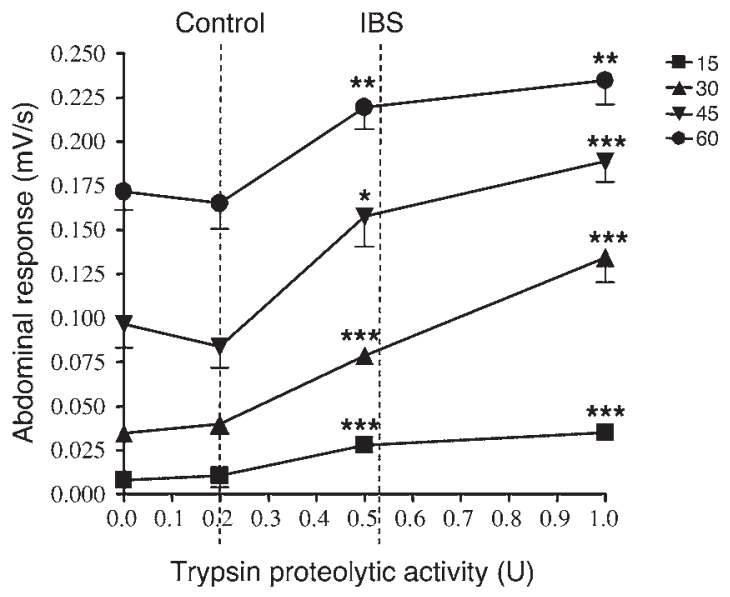


Table 1

Characteristics of patients from which biopsies were collected for culture media incubations

$\begin{array}{lccc}\text { Characteristic } & \text { Control }(\boldsymbol{n = 1 2}) & \text { IBS }(\boldsymbol{n}=\mathbf{1 8}) & \text { IBD }(\boldsymbol{n}=\mathbf{5}, \mathbf{4} \text { UC, } \mathbf{1} \text { CD }) \\ \text { Age (yr, range) } & 52(34-70) & 44(20-69) & 51(17-79) \\ \text { Sex (no., F/M) } & 8 / 4 & 16 / 2 & 2 / 3 \\ \text { Bowel movements } & & & 0 \\ \quad \text { Diarrhea } & 1^{\mathrm{A}} & 9 & 0 \\ \quad 1^{\mathrm{A}} & 4 & 0 \\ \quad \text { Alternating diarrhea/constipation } & 0 & 5 & 0 \\ \text { History of intestinal infection } & 0 & 1 & \text { 1 moderate, } \\ \text { Signs of inflammation } & 0 & 7^{\mathrm{B}} & \text { 3 moderate/severe, } \\ & & & \text { 1 severe }\end{array}$

Values are number of patients with characteristic unless otherwise indicated. AAcute episodes with no clinical evidence of IBS. BLymphocyte/plasma cell infiltration without crypt abscess; architectural distortion such as mucosal edema, superficial erosions, and crypt branching. CD, Crohn disease; UC, ulcerative colitis.

quantification of all samples. The trypsin/gusb mRNA ratio was calculated for each patient and then normalized to the average of the control patients. Primers used were as follows: trypsin, 382-bp product (forward 5'-CGCGTGTCCACCATCTCTCTGC-3' and reverse 5'-TTAGCTGTTGGCAGCTATGGTG-3') and gusb 81-bp product (forward 5'-CTCATTTGGAATTTTGCCGATT-3' and reverse 5'-CCGAGTGAAGATCCCCTTTTTA-3'). Cycling conditions were $94^{\circ} \mathrm{C}$ for $10 \mathrm{~min}$ utes, followed by $94^{\circ} \mathrm{C}$ for 30 seconds, $60^{\circ} \mathrm{C}$ for 30 seconds, and $72^{\circ} \mathrm{C}$ for 30 seconds for 30 cycles. Product identity was confirmed by sequencing. For tryptase, the PCR reaction was performed using a dual quantitative RT-PCR kit (Maxim Biotech Inc.). Two microliters of cDNA product was amplified in a final concentration of $35 \mu$ l of Master Mixture con-

were incubated in $2 \mathrm{ml}$ of $\mathrm{HBSS}$ at $37^{\circ} \mathrm{C}$ for 1 hour with a NF-кB inhibitor, BAY 11-7085 (20 $\mu \mathrm{M}$; BIOMOL) (14). For all experiments, supernatant volumes were standardized to the weight of incubated biopsies and not to supernatant protein, as protein content was below the detection threshold in biopsy supernatants. After incubation, biopsies were snap frozen in TRIzoL (Invitrogen) for further RT-PCR analysis. Other biopsies not used for incubations were fixed in Zamboni's solution overnight at $4^{\circ} \mathrm{C}$ for analyses of mast cells and tryptase immunoreactivity. Separate biopsies were obtained for routine histopathology performed by a histopathologist blinded to the patient's inclusion in the study. For the IBS patients, classification of inflamed versus noninflamed tissues was based on the report of the histopathologist on the presence of lymphocyte/plasma cell infiltration and architectural distortion, as described in Tables 1 and 2. This overlap between IBS and microscopic colitis is consistent with previously reported population-based studies of IBS patients (61). For IBD patients, histological evidence of IBD was also determined by a histopathologist.

Colonic washes were performed by instillation of $30 \mathrm{ml}$ of a saline solution $(0.9 \% \mathrm{NaCl})$, though the endoscope, into the patient's rectum (Table 2 ). The saline solution was left in the rectum lumen for 30 seconds and was then aspirated, filtered with a $0.2-\mu \mathrm{m}$ syringe filter (Acrodisc; Pall Corp.), and frozen. Collection of biopsies from these patients was performed as described above from nonwashed mucosa.

Proteinase activity assay. Trypsin-like activity was determined using a Fluoroskan Ascent microplate fluorometer (Thermo Scientific) as previously described (47) (excitation, $355 \mathrm{~nm}$; emission, $460 \mathrm{~nm}$ ). The hydrolysis rate was measured at room temperature over a 20 -minute period following addition of a biopsy supernatant sample $(5 \mu \mathrm{l})$ or colonic washes $(5 \mu \mathrm{l})$ to $200 \mu \mathrm{l}$ of substrate solution (Boc-Gln-Ala-Arg- AMC [75 $\mu \mathrm{M}]$ in Tris $[50 \mu \mathrm{M}], \mathrm{CaCl}_{2}[20 \mu \mathrm{M}]$ buffer, $\left.\mathrm{pH} 7.4\right)$ and was standardized to the rate generated by known concentrations of trypsin and normalized as described above to the weight of the biopsy. The proteolytic activity of the colonic washes was normalized to the protein concentration determined with a Micro BCA Protein Assay Kit (Pierce Biotechnologies). FUT-175 was added to biopsy supernatants at a final concentration of $50 \mu \mathrm{g} / \mathrm{ml}$.

RT-PCR for trypsin and tryptase. Upon thawing, biopsies were homogenized in TRIzoL and RNA extracted according to the manufacturer's guidelines. For trypsin, RNA was quantified and $2 \mu$ g reverse transcribed using TaqMan reverse transcription reagents (Applied Biosystems) according to the manufacturer's instructions. The PCR reaction was sampled at 15, 20, 25, 30 , and 35 cycles. Only at 30 cycles could products be seen for both trypsin and the $\beta$-glucuronidase (gusb) control. This cycle number was used for taining dNTPs and the primers for tryptase and GADPH (Maxim Biotech Inc.), and $1 \mathrm{U}$ of Taq polymerase (Maxim Biotech Inc.) in a total volume of $50 \mu \mathrm{l}$. For each sample, GADPH was coamplified as an internal control of tryptase amplification. Aliquots of PCR reactions (approximately $20 \mu \mathrm{l}$ ) were normalized to the equivalent signals from the GADPH mRNA and separated by electrophoresis through $1 \%$ agarose gels containing $0.2 \mu \mathrm{g} / \mathrm{ml}$ ethidium bromide. Gels were photographed and analyzed by densitometry using Bio-Rad imaging software Quantity One 4.6.2. Tryptase mRNA expression is presented as a ratio to GADPH.

Western blotting for trypsin, tryptase, $\alpha$-1-antiproteinase, and enterokinase. Proteins were precipitated from biopsy supernatants with 10\% TCA and resuspended with $30 \mu \mathrm{l}$ of HBSS (Sigma-Aldrich) and $30 \mu \mathrm{l}$ of Laemmli sample loading buffer (Sigma-Aldrich). Equal amounts (standardized according to biopsy tissue weight) of each supernatant were subjected to $10 \%$ SDS-PAGE and then electrotransferred onto a 0.45 - $\mathrm{mm}$ nitrocellulose membrane. Membranes were blocked with Tris-buffered saline with $5 \%$ milk blocking solution and then incubated overnight at $4{ }^{\circ} \mathrm{C}$ with the primary antibodies. We used a 1/5,000 dilution for the trypsin antibody (Chemicon International), 1/1,000 for the tryptase antibody (Chemicon International), $1 / 1,000$ for the $\alpha$-1-antiproteinase antibody (Cedarlane Laboratories Ltd.), and $2.5 \mu \mathrm{g} / \mathrm{ml}$ for the anti-enterokinase antibody (R\&D

\section{Table 2}

Characteristics of patients from which rectal lavages and/or biopsies were collected for proteolytic activity assessment and incubations with the NF-кB inhibitor BAY 11-7085

\begin{tabular}{|c|c|c|}
\hline Characteristic & Control $(n=10)$ & IBS $(n=9)$ \\
\hline Age (yr, range) & $46(31-63)$ & $43(22-58)$ \\
\hline Sex (no., F/M) & $7 / 3$ & $8 / 1$ \\
\hline \multicolumn{3}{|l|}{ Bowel movements } \\
\hline Diarrhea & 0 & $4^{A}$ \\
\hline Constipation & 0 & 2 \\
\hline Alternating diarrhea/constipation & 0 & $3^{A}$ \\
\hline History of intestinal infection & 0 & 0 \\
\hline Signs of inflammation ${ }^{B}$ & 0 & $1^{\mathrm{B}}$ \\
\hline
\end{tabular}


Systems). After washing, a secondary antibody (Jackson ImmunoResearch Laboratories Inc.) diluted 1/50,000 for trypsin and tryptase, 1/20,000 for enterokinase (donkey anti-mouse IgG or AffiniPure donkey anti-goat), or $1 / 20,000$ for $\alpha$-1-antitrypsin was added for 1 hour at room temperature. The membrane was incubated for 1-5 minutes with ECL-WB detection reagents (Amersham Biosciences). Films were scanned and the bands analyzed by densitometry using Bio-Rad imaging software Quantity One 4.6.2. Tryptase and trypsin protein amounts were expressed in pixels, representative of the optic density of detected bands.

Mast cells and tryptase immunoreactivity. Biopsy samples were fixed by immersion in Zamboni's fixative overnight at $4^{\circ} \mathrm{C}$. After washing in PBS, they were cryoprotected in PBS containing $20 \%$ sucrose. Sections were cut on a cryostat $(12 \mu \mathrm{m})$ and mounted on polylysine-coated slides. Sections were then rinsed in $0.125 \mathrm{~N} \mathrm{HCl}$ and stained with Alcian blue (1\% Alcian blue [BDH Chemicals Ltd.] in $0.7 \mathrm{~N} \mathrm{HCl}$ ) for 60 minutes at room temperature. They were differentiated in $0.125 \mathrm{~N} \mathrm{HCl}$ and washed in distilled water, dehydrated, and mounted. Blue-stained mucosal mast cells were counted using a Zeiss Axioplan microscope, and data are presented as cells per $\times 10$ objective field by an observer blinded to the patient groups. Data are presented as mean \pm SEM. Statistical comparison was made using a Mann-Whitney $U$ test.

For the assessment of tryptase immunoreactivity, sections were incubated for 48 hours at $4{ }^{\circ} \mathrm{C}$ in primary mouse anti-human tryptase monoclonal (clone G3, 1:1,000; Chemicon International; Millipore) (17). After incubation, sections were washed 3 time in PBS containing $0.1 \%$ Triton X-100 and incubated in secondary donkey anti-mouse conjugated to CY3 (1:100; Jackson ImmunoResearch Laboratories Inc.) for 1 hour at room temperature. They were then washed again as described above and mounted in bicarbonate-buffered glycerol. Immunoreactive cells were counted using a Zeiss Axioplan microscope, and data are presented as cells per $\times 40$ objective field by an observer blinded to the patient groups. Cells were counted in 3 random, nonoverlapping fields in 3 sections per patient in the lamina propria and epithelium, only if they displayed a prominent unlabeled nucleus. No cell fragments were included in the counts. In some sections, it was apparent that immunoreactivity was associated with degranulating mast cells. We did not count the degree of degranulation, since quantification of noncellular immunoreactivity was not readily accomplished. Cell counts were compared with a Mann-Whitney $U$ test.

Animals. Male C57BL/ 6 mice (6-8 weeks old) were obtained from Charles River Laboratories, and $\mathrm{PAR}_{2}{ }^{-/-}$mice originally from Johnson \& Johnson Research Pharmaceutical together with wild-type littermates were bred at the University of Calgary Animal Care facility. Mice were housed in transparent plastic cages with free access to food and water, in rooms with a 12-hour light/12-hour dark cycle. All experiments were approved by the Animal Care Committee of the University of Calgary and were performed in accordance with the guidelines of the Canadian Council on Animal Care. Mice were used for somatic and visceral nociception experiments, as well as for DRG neuron isolation.

$\mathrm{Ca}^{2+}$ imaging in sensory neurons. DRGs were rinsed in cold DMEM and incubated in DMEM containing $0.5 \mathrm{mg} / \mathrm{ml}$ trypsin, $1 \mathrm{mg} / \mathrm{ml}$ collagenase, and $0.1 \mathrm{mg} / \mathrm{ml}$ DNAse I (all from Sigma-Aldrich) for 45-60 minutes at $37^{\circ} \mathrm{C}(39,48)$. Soybean trypsin inhibitor (SBTI) (Sigma-Aldrich) was added to neutralize trypsin, and the suspension was centrifuged at about $1,000 \mathrm{~g}$ for 1 minute. Neurons in the pellet were suspended in DMEM containing $10 \%$ FBS, $2 \mathrm{mM}$ glutamine, $1 \mathrm{mg} / \mathrm{ml}$ penicillin/streptomycin, and DNAse I (all from Sigma-Aldrich). Cells were plated in Matrigel-treated glass-bottom Petri dishes (35-mm diameter; MatTek Corp.) and recovered with the complete culture media (described above). Calcium assay was performed 72 hours later after visual inspection of the cells, as previously described $(39,48)$. The Petri dishes were washed twice with HBSS pH 7.4 supple- mented with $0.1 \%$ BSA. The cells were then incubated for 45-60 minutes at $37^{\circ} \mathrm{C}$ in the same solution to which $3-5 \mu \mathrm{M}$ of Fluo3-AM (Invitrogen) was added. After the incubation, the Petri dishes were washed twice again with the assay buffer $\left(280 \mathrm{mM} \mathrm{CaCl}_{2}, 150 \mathrm{mM} \mathrm{NaCl}, 20 \mathrm{mM}\right.$ HEPES, 10 $\mathrm{mM}$ dextrose, $3 \mathrm{mM} \mathrm{KCl}, 250 \mu \mathrm{M}$ sulfinpyrazone, $\mathrm{pH} 7.4$ ), of which $2 \mathrm{ml}$ was left in each Petri dish. Cells were observed using a Wide-Field Fluorescence Olympus IX-70 Microscope and an LCPlan FL $\times 40$ objective. A kinetic of 30 pictures in 90 seconds was performed: neurons were exposed to control or IBS biopsy supernatant $(100 \mu \mathrm{l})$ after the fifth picture; the first 5 pictures were used to determine the baseline by fluorescence measurement at 460-490 $\mathrm{nm}$ excitation and $515 \mathrm{~nm}$ emission in individual cells, using the acquisition program OpenLab software (Agilent Technologies). The results were expressed as the ratio between the highest fluorescence measurement and the baseline. $\left[\mathrm{Ca}^{2+}\right]_{i}$ was calculated using the equation $\left[\mathrm{Ca}^{2+}\right]_{\mathrm{i}}=K_{d}\left(F-F_{\min }\right) /\left(F_{\max }-F\right)$, where $K_{d}$ is the dissociation constant of the $\mathrm{Ca}^{2+}-$ fluo- 3 complex $(400 \mathrm{nM})$, and $F$ represents the fluorescence intensity of the cells expressed as the ratio between the highest fluorescence measurement and the baseline. $F_{\max }$ represents the maximum fluorescence (obtained by treating cells with $10 \mu \mathrm{M}$ calcium ionophore A23187), and $F_{\min }$ corresponds to the minimum fluorescence (obtained from ionophore-treated cells in the presence of $3 \mathrm{mM} \mathrm{EGTA)}$. The fluorescence intensities were expressed as the increase in fluorescence with respect to baseline fluorescence intensity before stimulation (62). The experiments were repeated 3 times per biopsy. In a second set of experiments, neurons were treated with IBS supernatant preincubated for 10 minutes with the serine protease inhibitor (FUT-175, $50 \mu \mathrm{g} / \mathrm{ml}$ ).

Somatic nociception. Supernatants from IBS and control patient biopsy incubations or saline were injected into the mouse paw under light halothane anesthesia and in a total volume of $25 \mu$. Nociceptive responses to thermal stimulation were evaluated by measuring withdrawal latency after exposure of the mouse paw to radiant heat stimulus applied by a plantar test apparatus (Ugo Basile) (13). Nociceptive responses to mechanical stimulation were performed using von Frey monofilaments of different bending forces $(0.07 \mathrm{~g}$ for the 3.61 filament, $0.692 \mathrm{~g}$ for the 3.84 filament, and $1.202 \mathrm{~g}$ for the 4.08 filament) as previously described $(13,63,64)$. The minimum force required to elicit a reproducible withdrawal reflex on 3 applications of the von Frey filament to the plantar surface of the hindpaw was recorded as the mechanical nociceptive score. Scores were assigned based on the animal's response: 0 = no movement; 1 = removal of the paw; 2 = removal of the paw and vocalization or licking or holding of the paw. The score was expressed as a percentage of the maximal score for the 3 applications. Median and range are represented for percentage of maximal nociceptive score. Thermal and mechanical nociceptive responses were measured in basal conditions (in naive animals before any treatment) and hourly after the intraplantar injections. Each supernatant was injected into the paw of 6 different mice, and the mean of the nociceptive responses for each supernatant is presented in Figure 5.

Colorectal distention and electromyography recording. Mice were anaesthetized by xylazine, ketamine, and saline (1:20:29 ratio, intraperitoneally). Three groups of electrodes (Bioflex insulated wire AS631; Cooner Wire) were implanted in the abdominal external oblique musculature. Electrodes were exteriorized at the back of the neck and protected by a plastic tube attached to the skin. Colorectal distension was performed 5 days after the electrode implantation, as previously described (65). Mice were placed in plastic tunnels (3-cm diameter, 10-cm length) in which they could not move, escape, or turn around. They were accustomed to this procedure for 1 day before rectal distension, to minimize stress reactions during experiments. The wires were connected to a Bio Amp (ADInstruments) itself connected to a PowerLab (ADInstruments) used as an electromyogram acquisition system using Chart 5 software (ADInstruments). Colorectal distension was then 
performed in a stepwise fashion. The balloon (10.5-mm diameter) used for distensions was an arterial embolectomy probe (LeMaitre Embolectomy Catheter 4F; LeMaitre Vascular). Rectal distension was performed by insertion of the balloon into the colon at $5 \mathrm{~mm}$ proximal to the rectum. The balloon was then inflated in a stepwise fashion from 0 to $60 \mathrm{mmHg}$ in 15 -mmHg increments. Ten-second distensions were performed in triplicate at pressures of $15,30,45$, and $60 \mathrm{mmHg}$ with 5 -minute intervals. The balloon, connected to a manometer and a syringe, was filled with tepid water in order to avoid temperature contrast. To detect possible leakage, the volume of water introduced into the balloon was verified by complete removal at the end of the distension period.

Biopsy supernatants from control or IBS patients (100 $\mu \mathrm{l})$ were administrated intracolonically to mice under light halothane anesthesia. At set times after biopsy sample administration (0, 3, 6, 10, and 24 hours), a series of rectal distentions were performed to generate a pressure-response curve. In a second set of experiments, 3 groups of 17 mice had identical intracolonic instillation of IBS supernatants, and colorectal distension was performed, once before the administration and again 6 hours after. The first group received the IBS biopsy supernatant preincubated with the serine protease inhibitor (FUT-175, $50 \mu \mathrm{g} / \mathrm{ml}$ ) for 10 minutes before the intracolonic administration. The second group was pretreated with a $\mathrm{PAR}_{2}$ antagonist ( $4 \mathrm{mg} /$ mouse; given intraperitoneally) 30 minutes before the intracolonic administration, as previously described (19). The $\mathrm{PAR}_{2}$ antagonist was obtained from T. Hembrough (EntreMed Inc., Rockville, Maryland, USA), and its pharmacological characteristics were published in Kelso et al. (19). For the last group, $P A R_{2}{ }^{-{ }^{-}}$mice were used and the intracolonic administration of IBS biopsy supernatant was performed. In a last set of experiments, 4 groups of 10 mice were administrated intracolonically $100 \mu \mathrm{l}$ of trypsin (Sigma-Aldrich) at $0,0.2,0.5$, and $1.0 \mathrm{U}$ respectively; colorectal distension was performed 6 hours after administration. The trypsin was dissolved in saline solution ( $0.9 \%$ sodium chloride).
Statistics. Data are presented as mean \pm SEM except for nociceptive scores. Analyses were performed using GraphPad Prism 3.0 software. All data were normally distributed. Between-group comparisons were performed by Student's unpaired 2-tailed $t$ test. Multiple comparisons within groups were performed by repeated-measures 1-way ANOVA, followed by Tukey's procedure. The Mann-Whitney $U$ test was used for the comparison of nociceptive scores in IBS-injected mice compared with control-injected mice. Statistical significance was accepted at $P<0.05$.

\section{Acknowledgments}

Nicolas Cenac is supported by a Canadian Association of Gastroenterology/Crohn's and Colitis Foundation (CCFC) of Canada fellowship. Nathalie Vergnolle is an Alberta Heritage Foundation for Medical Research (AHFMR) Scholar and a Canadian Institute of Health Research (CIHR) New Investigator. Keith A. Sharkey is an AHFMR Medical Scientist and CCFC Chair in IBD research. Funding for this research was provided by the CIHR (to N. Vergnolle and K.A. Sharkey), NIH grants (DK57480 and DK43207 to N.W. Bunnett), Forschungsgemeinschaft (STE 1014/2-1 to M. Steinhoff), IZKF Munster (to M. Steinhoff), and Sonderforschungsbereich 293A14 (to M. Steinhoff). We thank Winnie Ho for skilled assistance.

Received for publication July 25, 2006, and accepted in revised form December 5, 2006.

Address correspondence to: Nathalie Vergnolle, Department of Pharmacology and Therapeutics, Faculty of Medicine, University of Calgary, 3330 Hospital Drive NW, Calgary, Alberta, T2N4N1, Canada. Phone: (403) 220-4588; Fax: (403) 210-8195; E-mail: nvergnol@ucalgary.ca.
1. Johanson, J.F. 2004. Options for patients with irritable bowel syndrome: contrasting traditional and novel serotonergic therapies. Neurogastroenterol. Motil. 16:701-711.

2. Camilleri, M. 2005. Mechanisms in IBS: something old, something new, something borrowed. Neurogastroenterol. Motil. 17:311-316.

3. Cremonini, F., and Talley, N.J. 2005. Treatments targeting putative mechanisms in irritable bowel syndrome. Nat. Clin. Pract. Gastroenterol. Hepatol. 2:82-88.

4. Schwetz, I., Bradesi, S., and Mayer, E.A. 2004. The pathophysiology of irritable bowel syndrome. Minerva Med. 95:419-426.

5. Bercik, P., Verdu, E.F., and Collins, S.M. 2005. Is irritable bowel syndrome a low-grade inflammatory bowel disease? Gastroenterol. Clin. North Am. 34:235-245, vi-vii.

6. Collins, S.M., Piche, T., and Rampal, P. 2001. The putative role of inflammation in the irritable bowel syndrome. Gut. 49:743-745.

7. Spiller, R.C. 2004. Inflammation as a basis for functional GI disorders. Best Pract. Res. Clin. Gastroenterol. 18:641-661.

8. Andrews, C.N., and Shaffer, E.A. 2004. Simmering innards: does irritable bowel syndrome have an immunological basis? Can. J. Gastroenterol. 18:601-603.

9. Spiller, R.C. 2003. Postinfectious irritable bowel syndrome. Gastroenterology. 124:1662-1671.

10. Quigley, E.M. 2005. Irritable bowel syndrome and inflammatory bowel disease: interrelated diseases? Chin. J. Dig. Dis. 6:122-132.

11. Mertz, H., Naliboff, B., Munakata, J., Niazi, N., and Mayer, E.A. 1995. Altered rectal perception is a biological marker of patients with irritable bowel syndrome. Gastroenterology. 109:40-52.
12. Coelho, A.M., Vergnolle, N., Guiard, B., Fioramonti, J., and Bueno, L. 2002. Proteinases and proteinase-activated receptor 2: a possible role to promote visceral hyperalgesia in rats. Gastroenterology. 122:1035-1047.

13. Vergnolle, N., et al. 2001. Proteinase-activated receptor- 2 and hyperalgesia: a novel pain pathway. Nat. Med. 7:821-826.

14. Lawrence, T., Gilroy, D.W., Colville-Nash, P.R., and Willoughby, D.A. 2001. Possible new role for NF-kappaB in the resolution of inflammation. Nat. Med. 7:1291-1297.

15. Miller, H.R., and Pemberton, A.D. 2002. Tissue-specific expression of mast cell granule serine proteinases and their role in inflammation in the lung and gut. Immunology. 105:375-390.

16. Wong, G.W., Yang, Y., Yasuda, S., Li, L., and Stevens, R.L. 2003. Mouse mast cells express the tryptic protease neuropsin/Prss19. Biochem. Biophys. Res. Commun. 303:320-325.

17. Irani, A.A., Schechter, N.M., Craig, S.S., DeBlois, G., and Schwartz, L.B. 1986. Two types of human mast cells that have distinct neutral protease compositions. Proc. Natl. Acad. Sci. U. S. A. 83:4464-4468.

18. Light, A., and Janska, H. 1989. Enterokinase (enteropeptidase): comparative aspects. Trends Biochem. Sci. 14:110-112.

19. Kelso, E.B., et al. 2005. Therapeutic promises of PAR2 antagonism in joint inflammation. J. Pharmacol. Exp. Ther. 316:1017-1024.

20. Vergnolle, N. 2005. Clinical relevance of proteinaseactivated receptors in the gut. Gut. 54:867-874.

21. Cuffe, J.E., et al. 2002. Basolateral PAR-2 receptors mediate $\mathrm{KCl}$ secretion and inhibition of $\mathrm{Na}(+)$ absorption in the mouse distal colon. J. Physiol. 539:209-222.

22. Green, B.T., Bunnett, N.W., Kulkarni-Narla, A.,
Steinhoff, M., and Brown, D.R. 2000. Intestinal type 2 proteinase-activated receptors: expression in opioid-sensitive secretomotor neural circuits that mediate epithelial ion transport. J. Pharmacol. Exp. Ther. 295:410-416.

23. Mall, M., et al. 2002. Activation of ion secretion via proteinase-activated receptor-2 in human colon. Am. J. Physiol. Gastrointest. Liver Physiol. 282:G200-G210.

24. Vergnolle, N., et al. 1998. Proteinase-activated receptor 2 (PAR2)-activating peptides: identification of a receptor distinct from PAR2 that regulates intestinal transport. Proc. Natl. Acad. Sci. U. S. A. 95:7766-7771.

25. Cenac, N., et al. 2002. Induction of intestinal inflammation in mouse by activation of proteinaseactivated receptor-2. Am. J. Pathol. 161:1903-1915.

26. Cenac, N., et al. 2004. PAR2 activation alters colonic paracellular permeability in mice via IFNgamma-dependent and-independent pathways. J. Physiol. 558:913-925.

27. Barau, E., and Dupont, C. 1990. Modifications of intestinal permeability during food provocation procedures in pediatric irritable bowel syndrome. J. Pediatr. Gastroenterol. Nutr. 11:72-77.

28. Spiller, R.C., et al. 2000. Increased rectal mucosal enteroendocrine cells, T lymphocytes, and increased gut permeability following acute Campylobacter enteritis and in post-dysenteric irritable bowel syndrome. Gut. 47:804-811.

29. Ait-Belgnaoui, A., Bradesi, S., Fioramonti, J., Theodorou, V., and Bueno, L. 2005. Acute stress-induced hypersensitivity to colonic distension depends upon increase in paracellular permeability: role of myosin light chain kinase. Pain. 113:141-147.

30. Kawabata, A., et al. 2001. In vivo evidence that protease-activated receptors 1 and 2 modulate gastrointestinal transit in the mouse. Br. J. Pharmacol. 
133:1213-1218.

31. Mule, F., Baffi, M.C., and Cerra, M.C. 2002. Dual effect mediated by protease-activated receptors on the mechanical activity of rat colon. Br. J. Pharmacol. 136:367-374.

32. Zhao, A., and Shea-Donohue, T. 2003. PAR-2 agonists induce contraction of murine small intestine through neurokinin receptors. Am. J. Physiol. Gastrointest. Liver Physiol. 285:G696-G703.

33. Kirkup, A.J., Jiang, W., Bunnett, N.W., and Grundy, D. 2003. Stimulation of proteinase-activated receptor 2 excites jejunal afferent nerves in anaesthetised rats. J. Physiol. 552:589-601.

34. Reed, D.E., et al. 2003. Mast cell tryptase and proteinase-activated receptor 2 induce hyperexcitability of guinea pig submucosal neurons. J. Physiol. 547:531-542.

35. Nguyen, C., et al. 2003. Proteinase-activated receptor-2-induced colitis is mediated by a neurogenic mechanism. Can. J. Physiol. Pharmacol. 81:920-927.

36. Kim, J.A., et al. 2003. Expression of protease-activated receptor 2 in ulcerative colitis. Inflamm. Bowel Dis. 9:224-229.

37. Grundy, D. 2004. What activates visceral afferents? Gut. 53(Suppl. 2):ii5-ii8.

38. Bueno, L., and Fioramonti, J. 2002. Visceral perception: inflammatory and non-inflammatory mediators. Gut. 51(Suppl. 1):i19-i23.

39. Amadesi, S., et al. 2004. Protease-activated receptor 2 sensitizes the capsaicin receptor transient receptor potential vanilloid receptor 1 to induce hyperalgesia. J. Neurosci. 24:4300-4312.

40. Dai, Y., et al. 2004. Proteinase-activated receptor 2mediated potentiation of transient receptor potential vanilloid subfamily 1 activity reveals a mechanism for proteinase-induced inflammatory pain. J. Neurosci. 24:4293-4299.

41. Barbara, G., et al. 2002. Expression and release of mast cell tryptase in irritable bowel syndrome (IBS) [abstract]. Gastroenterology. 122:A276.

42. Barbara, G., et al. 2004. Activated mast cells in proximity to colonic nerves correlate with abdominal pain in irritable bowel syndrome. Gastroenterology. 126:693-702.

43. Weston, A.P., Biddle, W.L., Bhatia, P.S., and Miner, P.B., Jr. 1993. Terminal ileal mucosal mast cells in irritable bowel syndrome. Dig. Dis. Sci. 38:1590-1595.
44. Corvera, C.U., et al. 1999. Thrombin and mast cell tryptase regulate guinea-pig myenteric neurons through proteinase-activated receptors-1 and -2 . J. Physiol. (Lond.). 517:741-756.

45. Gao, C.Y., et al. 2002. Serine proteases excite myenteric neurons through protease-activated receptors in guinea pig small intestine. Gastroenterology. 123:1554-1564.

46. Cottrell, G.S., Amadesi, S., Grady, E.F., and Bunnett, N.W. 2004. Trypsin IV: a novel agonist of protease-activated receptors 2 and 4. J. Biol. Chem. 279:13532-13539.

47. Hansen, K.K., et al. 2005. A major role for proteolytic activity and proteinase-activated receptor-2 in the pathogenesis of infectious colitis. Proc. Natl. Acad. Sci. U. S. A. 102:8363-8368.

48. Steinhoff, M., et al. 2000. Agonists of proteinaseactivated receptor 2 induce inflammation by a neurogenic mechanism. Nat. Med. 6:151-158.

49. Malinen, E., et al. 2005. Analysis of the fecal microbiota of irritable bowel syndrome patients and healthy controls with real-time PCR. Am. J. Gastroenterol. 100:373-382.

50. Maukonen, J., et al. 2006. Prevalence and temporal stability of selected clostridial groups in irritable bowel syndrome in relation to predominant faecal bacteria. J. Med. Microbiol. 55:625-633.

51. Macfarlane, S.R., et al. 2005. The role of intracellular $\mathrm{Ca}^{2}$ in the regulation of proteinase-activated receptor- 2 mediated nuclear factor kappa B signalling in keratinocytes. Br. J. Pharmacol. 145:535-544.

52. Uehara, A., Muramoto, K., Takada, H., and Sugawara, S. 2003. Neutrophil serine proteinases activate human nonepithelial cells to produce inflammatory cytokines through protease-activated receptor 2. J. Immunol. 170:5690-5696.

53. Lin, C.H., et al. 2006. c-Src mediates thrombininduced NF-kappaB activation and IL-8/CXCL8 expression in lung epithelial cells. J. Immunol. 177:3427-3438.

54. Uehara, A., et al. 2005. Arginine-specific gingipains from Porphyromonas gingivalis stimulate production of hepatocyte growth factor (scatter factor) through protease-activated receptors in human gingival fibroblasts in culture. J. Immunol. 175:6076-6084.

55. Hiramitsu, T., et al. 2006. Intercellular adhesion molecule- 1 mediates the inhibitory effects of hyaluronan on interleukin-1beta-induced matrix metalloproteinase production in rheumatoid synovial fibroblasts via down-regulation of NF-kappaB and p38. Rheumatology (Oxford). 45:824-832.

56. Philip, S., Bulbule, A., and Kundu, G.C. 2001. Osteopontin stimulates tumor growth and activation of promatrix metalloproteinase- 2 through nuclear factor-kappa B-mediated induction of membrane type 1 matrix metalloproteinase in murine melanoma cells. J. Biol. Chem. 276:44926-44935.

57. Philip, S., Bulbule, A., and Kundu, G.C. 2004. Matrix metalloproteinase-2: mechanism and regulation of NF-kappaB-mediated activation and its role in cell motility and ECM-invasion. Glycoconj.J. 21:429-441.

58. Linden, D.R., Manning, B.P., Bunnett, N.W., and Mawe, G.M. 2001. Agonists of proteinase-activated receptor 2 excite guinea pig ileal myenteric neurons. Eur. J. Pharmacol. 431:311-314.

59. Thompson, W.G. 1999. The road to Rome. Gut. 45(Suppl. 2):II80.

60. Thompson, W.G., Irvine, E.J., Pare, P., Ferrazzi, S., and Rance, L. 2002. Functional gastrointestinal disorders in Canada: first population-based survey using Rome II criteria with suggestions for improving the questionnaire. Dig. Dis. Sci. 47:225-235.

61. Limsui, D., et al. 2005. Symptomatic overlap between irritable bowel syndrome and microscopic colitis [abstract]. Gastroenterology. 128:A330.

62. Mishra, D.P., Pal, R., and Shaha, C. 2006. Changes in cytosolic $\mathrm{Ca}_{2}{ }^{+}$levels regulate $\mathrm{Bcl}-\mathrm{xS}$ and $\mathrm{Bcl}-\mathrm{xL}$ expression in spermatogenic cells during apoptotic death. J. Biol. Chem. 281:2133-2143.

63. Asfaha, S., Brussee, V., Chapman, K., Zochodne, D.W., and Vergnolle, N. 2002. Proteinase-activated receptor-1 agonists attenuate nociception in response to noxious stimuli. Br. J. Pharmacol. 135:1101-1106.

64. Boisse, L., Spencer, S.J., Mouihate, A., Vergnolle, N., and Pittman, Q.J. 2005. Neonatal immune challenge alters nociception in the adult rat. Pain. 119:133-141.

65. Larsson, M., Arvidsson, S., Ekman, C., and Bayati, A. 2003. A model for chronic quantitative studies of colorectal sensitivity using balloon distension in conscious mice - effects of opioid receptor agonists. Neurogastroenterol. Motil. 15:371-381. 